\title{
Skill Transference of a Probe-Tube Placement Training Simulator
}

DOI: $10.3766 /$ jaaa. 18054

\author{
Robert W. Koch* \\ Hasan Saleh $\dagger$ \\ Paula Folkeard $\neq$ \\ Sheila Moodie $\dagger+$ \\ Conner Janeteas§ \\ Sumit K. Agrawal* $+\mathbb{\top} \| * *$ \\ Hanif M. Ladak* $* \mathbb{\Phi} \| * *$ \\ Susan Scollie $†$
}

\begin{abstract}
Background: Probe-tube placement is a necessary step in hearing aid verification which needs ample hands-on experience and confidence before performing in clinic. To improve the methods of training in probe-tube placement, a manikin-based training simulator was developed consisting of a 3D-printed head, a flexible silicone ear, and a mounted optical tracking system. The system is designed to provide feedback to the user on the depth and orientation of the probe tube, and the time required to finish the task. Although a previous validation study was performed to determine its realism and teachability with experts, further validation is required before implementation into educational settings.
\end{abstract}

Purpose: This study aimed to examine the skill transference of a newly updated probe-tube placement training simulator to determine if skills learned on this simulator successfully translate to clinical scenarios.

Research Design: All participants underwent a pretest in which they were evaluated while performing a probe-tube placement and real-ear-to-coupler difference (RECD) measurement on a volunteer. Participants were randomized into one of two groups: the simulator group or the control group. During a twoweek training period, all participants practiced their probe-tube placement according to their randomly assigned group. After two weeks, each participant completed a probe-tube placement on the same volunteer as a posttest scenario.

Study Sample: Twenty-five novice graduate-level student clinicians.

Data Collection and Analysis: Participants completed a self-efficacy questionnaire and an expert observer completed a questionnaire evaluating each participant's performance during the pre- and posttest sessions. RECD measurements were taken after placing the probe tube and foam tip in the volunteer's ear. Questionnaire results were analyzed through nonparametric $t$-tests and analysis of variance, whereas RECD results were analyzed using a nonlinear mixed model method.

Results: Results suggested students in the simulator group were less likely to contact the tympanic membrane when placing a probe tube, appeared more confident, and had better use of the occluding foam tip, resulting in more improved RECD measurements.

Conclusions: The improved outcomes for trainees in the simulator group suggest that supplementing traditional training with the simulator provides useful benefits for the trainees, thereby encouraging its usage and implementation in educational settings.

${ }^{*}$ School of Biomedical Engineering, Western University, London, ON, Canada; †School of Communication Sciences and Disorders, Western University, London, ON, Canada; †National Centre for Audiology, Faculty of Health Sciences, Western University, London, ON, Canada; §Cimetrix Solutions Inc., Oshawa, ON, Canada; $\uparrow$ Department of Medical Biophysics, Western University, London, ON, Canada; ||Department of Otolaryngology - Head and Neck Surgery, Western University, London, ON, Canada; ${ }^{\star \star}$ Department of Electrical and Computer Engineering, Western University, London, ON, Canada

Corresponding author: Robert W. Koch, Biomedical Engineering, Western University, London, ON, Canada N6A 3K7; Email: rkoch2@uwo.ca

Funding for this project was provided through an Ontario Graduate Scholarship (2016/2017) through a Natural Sciences and Engineering Research Council (NSERC) Canadian Graduate Scholarship-Masters (CGSM) and through an NSERC Discovery Grant. 
Key Words: education, hearing aids, probe-tube placement, real ear, simulation, skill transference, training

Abbreviations: ANOVA = analysis of variance; RECD = real-ear-to-coupler difference; SD = standard deviation; $\mathrm{TM}=$ tympanic membrane

\section{INTRODUCTION}

$\mathrm{H}$ earing aid verification is a recommended step in the fitting of hearing aids. International organizations for audiology require that hearing aids undergo verification by the prescribing clinician (Valente, 2006; BSA, 2007; CASLPO, 2016; ISO, 2001), with research continuing to support that fittings verified by a real-ear fit to prescribed targets provide better audibility to patients than fittings performed without verification (Aazh and Moore, 2007; Abrams et al, 2012; Leavitt and Flexer, 2012; Valente et al, 2017). However, some hearing aid fittings omit verification which increases the chances that they do not provide adequate audibility and/or provide overamplification for patients. Studies have found that hearing aids are not being accurately fitted to the target more than $50 \%$ of the time (Consumer Reports, 2009; McCreery et al, 2013; Leavitt et al, 2017). A recent study reported that hearing aid users wore hearing aids with deviations from NAL to NL2 targets of $>10 \mathrm{~dB}$ in $72 \%$ of fittings and errors of $>5 \mathrm{~dB}$ in $97.7 \%$ of fittings (Leavitt et al, 2017). This may be attributed to $>50 \%$ of providers that are not regularly conducting real-ear measurements (Bamford et al, 2001; Mueller, 2005; Mueller and Picou, 2010), resulting in deviations from targets in excess of $10 \mathrm{~dB}$ when manufacturer's first fit settings are used (Aazh et al, 2012). Similarly, McCreery et al (2013) found that deviations from targets in children's fittings were greater when real-ear measurement (via the real-earto-coupler difference [RECD]) was estimated from average values rather than individually measured. There is a gap that exists between best practice recommendations for verification of all hearing aid fittings and the actual use of real-ear hearing aid verification in clinical practice.

Many explanations for the lack of routine verification have been proposed such as the extra time required at fitting, cost of equipment, complexity of modern hearing aids, lack of self-confidence, and lack of training in the use of verification equipment (Mueller and Picou, 2010; Mueller, 2005; Jorgensen, 2016; Moodie, Rall, et al, 2016). Of all the suggested explanations, limitations in training can be directly addressed to increase confidence, skill, and competency in verification procedures. Clinicians responsible for the prescription of hearing aids must have knowledge of how to properly perform hearing aid verification, be provided with sufficient practice opportunities in executing these procedures, and exhibit competency, or they will be less likely to use real-ear verification within practice settings.

A key part of verification, which requires experience to perform effectively in clinic, is probe-tube placement. In 2016, Moodie et al found that $12 \%$ of pediatric audiologists who own real-ear measurement equipment do not perform real-ear measurements because of lack of confidence (Moodie, Rall, et al, 2016). A recent adultfocused survey indicated that $32 \%$ of audiologists in the United States sometimes (or less) performed probe-microphone measures with their adult patients (Anderson et al, 2018). Similarly, a survey by Mueller found that probe-tube placement was only performed approximately $40 \%$ of the time (Mueller and Picou, 2010). The insertion of a thin flexible probe tube into a patient's ear canal, along with the insertion of a foam tip/earmold, to occlude the ear canal for real-ear measurements is a complex task that requires specific training to achieve full competency in clinical practice. The main difficulty with probe-tube placement lies in placing the tube within the recommended $5 \mathrm{~mm}$ from the tympanic membrane (TM) (Dirks and Kincaid, 1987; Bagatto et al, 2006; Moodie, Pietrobon, et al, 2016; Vaisberg et al, 2016) and then ensuring no movement of the probe tube closer to, or further from, the TM after insertion of a hearing aid or foam eartip. If placed too close, the clinician risks contacting the TM of the patient, which may result in minor discomfort and a sound normally described as either scratching or a "thud." These unintended consequences are unpleasant for adults and may impact clinician-patient trust. If placed farther than $5 \mathrm{~mm}$ from the TM, standing waves will be introduced to the measurement causing incorrect measurements (Dirks and Kincaid, 1987; Pumford and Sinclair, 2001; Caldwell et al, 2006).

The traditional course instruction in probe-tube placement may include course readings and lectures on the anatomy of the ear canal, the acoustics of standing waves in the ear canal, recommended probe-tube placement procedures, troubleshooting, terminology, and normative data for real-ear measurements (Palmer, 1998). In present curricula, some schools also provide a laboratory environment in which students receive hands-on training, opportunity to practice on one another, and a practical examination. These training methods, however, may not be sufficient.

A common training method to provide additional hands-on preclinical experience is through simulation (Issenberg et al, 1999; Gaba, 2004; Kunkler, 2006; 
Wang et al, 2011; Brown, 2017). Although simulation has been evident in other medical fields, it has been used less frequently in audiology. Medical simulation allows for the practice of procedures in safe, controlled environments to prevent poor clinical performance. Simulation is an outlet in which novice clinicians can practice their procedures without the need for volunteers or instructors and receive validated feedback to better their performance. Through structured simulation and lesson plans, individuals can learn all aspects of their procedure with limited instructor time required. Simulation may, therefore, provide supplementary training over and above traditional practice methods.

A training simulator was developed at Western University, which aimed at providing audiology students with a method to practice probe-tube placement. The simulator allows students to receive real-time feedback of their probe-tube placement and to become comfortable with the procedure, and provides instructors with the ability to evaluate the students' performance before clinical placement. A previous validation study was performed evaluating the realism and training ability of the simulator, while outlining clear areas of improvement that needed attention before implementation into training programs (Koch et al, 2018). The results from this first study were encouraging; participants indicated that the simulator had high teaching value and provided an excellent opportunity for trainees to gain additional preclinical experience. All participants recommended implementation into educational settings once suggested improvements were made. These improvements have since been made to the simulator to improve its usability and effectiveness in training (Figure 1). A typical next step that is performed on medical training simulators is a skill transference study (Torkington et al, 2001; Laschinger et al, 2008; Dawe et al, 2014; Huang et al, 2015). A skill transference validation study aims to determine if skills learned on the simulator effectively translate to clinical application.

The objective of this study was to evaluate the skill transference of the probe-tube placement simulator by determining if the use of this simulator in preclinical scenarios could increase the competence and confidence of users in probe-tube placement and real-ear measurement in real-life clinical application.

\section{MATERIALS AND METHODS}

\section{Simulator}

Briefly, the simulator consisted of an artificial adultsized head with a pliable, realistic ear and a camera mounted within the head to measure the probe-tube insertion depth using an associated custom-developed software package. The original simulator has been previously described by Koch et al (2018) and used a

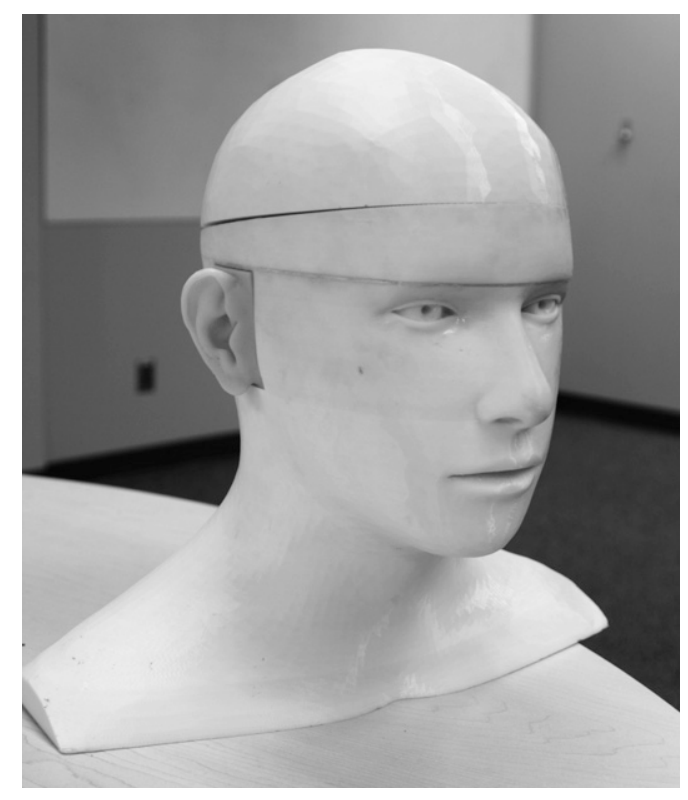

Figure 1. Image of the new probe-tube placement training simulator with a 3D-printed head and silicone ear model. The camera system is mounted inside the head to give the user real-time feedback on the placement of the probe inside the ear canal.

Styrofoam head that lacked realism as the first-generation prototype. Several improvements were made based on suggestions from the participants in the first validation study. The following improvements were only implemented on an adult model for the purposes of the present study. These updates incorporated a realistic 3D-printed head model, swappable silicone ears developed from computed tomography scans to represent variability in ear canal anatomy, and an improved mounted optical tracking system for tracking the location of the probe microphone inside the ear (Figure 1). Whereas the previous simulator used a directly 3Dprinted ear printed with a Stratasys Objet500 Connex3 $3 \mathrm{D}$ printer (Stratasys Ltd., Eden Prairie, MN), the improved silicone outer ear was created by printing the ear with a Lulzbot Taz 6 printer (Aleph Objects, Loveland, $\mathrm{CO}$ ) and using silicone to mold and cast the pinna and entrance to the canal. The casting and molding technique was used in this prototype as current 3D printing does not allow for the printing of highly flexible materials with complex structures. A silicone Shore value (hardness) of $2 \mathrm{~A}$ was selected by experts after experimenting with various material hardness properties. When this silicone pinna is inserted into the head, the entrance of the canal aligns with a 3D-printed transparent ear canal fastened to the head. The ear canal is printed with a Stratasys Objet500 Connex3 3D printer using a transparent material (VeroClearRGD810; Stratasys Ltd., Eden Prairie, MN) to allow for measurement of the probe-tube depth to the tenth of a millimeter from the tracking system. A high-resolution picture of an eardrum was attached to the end of the 
transparent ear canal to provide users with a realistic otoscopic image of the ear, as seen in Figure 2.

The previous Styrofoam head was replaced by a fully $3 D$-printed head and shoulders. The new head was printed out of polycarbonate-acrylonitrile butadiene styrene on a Stratasys Fortus $40 \mathrm{mc} 3 \mathrm{D}$ printer at a slice height of 0.010 inches. The existing camera system remained mounted inside the head model using a Microsoft LifeCam HD-3000 (Microsoft Corporation, Redmond, WA) connected to a typical laptop operating on a Windows operating system. The user interface, responsible for providing users with feedback metrics such as probe-to-TM distance and time to insert, was redesigned using OpenCV (OpenCV Team, 2018) and Qt (The Qt Company, Espoo, Finland). The simulator focused on two aspects of probetube placement, and therefore, two modes were developed in the program: (a) practice mode and (b) test mode. In practice mode, users were able to view a coronal image of the ear canal seen from an anterior position at any point during their practice, with an exact probe-to-TM distance to determine probe positioning inside the ear canal, whereas in test mode, this image and feedback were only available after the user had finished placing the probe tube. If at any point during an insertion in practice or test mode the user contacted the TM, a "grunt" audio stimulus was presented to alert the user of contact with the TM.

\section{Participants}

The study was approved by the Western University Health Research Ethics Board (HREB 110394). Partic-

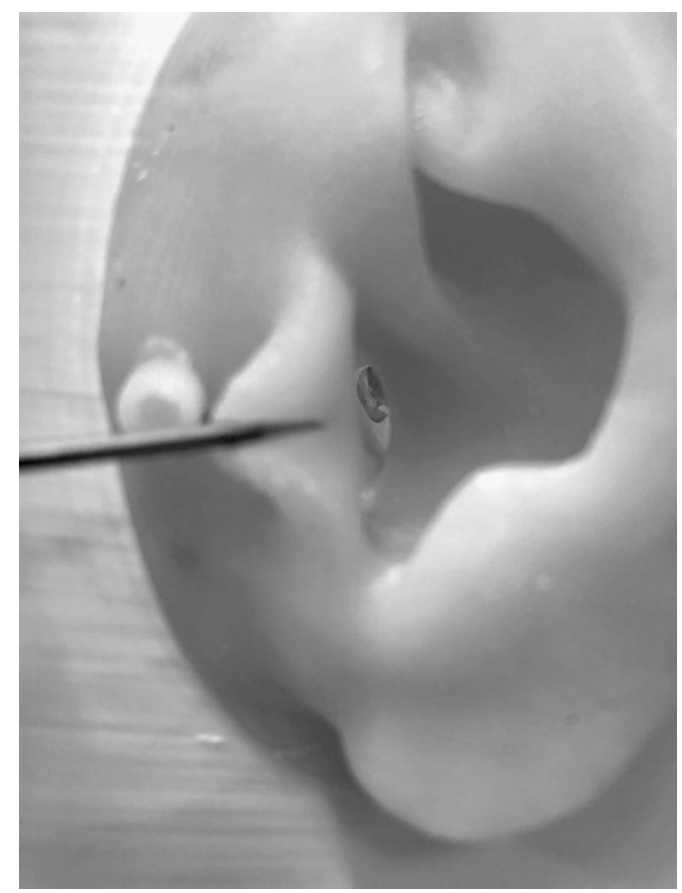

Figure 2. Image of the silicone ear model with the tragus moved aside showcasing the auricle, ear canal, and TM. ipants were recruited through a first-year graduatelevel audiology course that introduces procedures for the fitting of hearing aids. Twenty-five novice clinicians in this first-year class chose to participate. These students were composed of individuals in their second semester of their audiology program, with $<10$ hours of clinical experience. On beginning the study, participants reported having zero to three hours of experience in probe-tube placement.

\section{Protocol}

The study performed was a randomized controlled trial in which participants were placed into one of two groups: the control group or the simulator (treatment) group. The study consisted of three parts: (a) pretest evaluation, (b) training period, and (c) posttest evaluation.

\section{Pretest}

At the pretest evaluation (part 1), the participants had no knowledge of which group they were assigned to. During this pretest, both a volunteer and an expert evaluator (audiologist) were present in the room. The same volunteer and expert evaluator were present for all subsequent testing. To begin the pretest session, the participant completed the first half of the selfefficacy questionnaire (Table 1 , section A) regarding their confidence in performing a probe-tube placement and foam tip insertion. Following the survey, the participant prepared the equipment for an RECD measurement procedure (Audioscan Verifit VF1; Audioscan, Dorchester, ON, Canada) and inserted a probe tube into the volunteer's ear. After they were satisfied with their probe-tube placement (using otoscopy to verify placement), the participant inserted a foam tip connected to an RECD transducer and measured the volunteer's RECD values. RECD measurements were exported to a data file for further analysis. Only one probe-tube placement and RECD measurement were recorded per participant. Following this measurement, the participant completed the self-efficacy survey (Table 1, section B), the expert filled out their survey assessing the participant's performance (Table 1, section C), and the participant was informed of their randomly assigned group.

\section{Training Period}

The training period (part 2) consisted of two weeks in which the participants were instructed to practice as much or as little as they would for a practical examination. The training period was restricted to two weeks as not to interfere with coursework and to allow the control group enough time to practice with the simulator if they desired before their program's practical examination. 
Table 1. The Questionnaire Used to Evaluate the Participant's Skill and Confidence in this Real-Ear Measure

\begin{tabular}{lrl}
\hline Section & No. & \\
\hline A & 1 & You can place the probe within $5 \mathrm{~mm}$ from the TM. \\
& 2 & You can place the probe without contacting the TM. \\
& 3 & You can insert the foam tip without affecting the probe's location. \\
B & 4 & Participant self-evaluation after placement \\
& 5 & How certain are you the probe was within $5 \mathrm{~mm}$ of the TM?
\end{tabular}

Questions 1-3 were completed by the participant before the pre- or posttest session. Questions 4 and 5 were completed by the participant after the placement. Questions 6-10 were completed by the expert evaluator after the participants had completed their placement and measurements.

Each participant was given a practice diary to log the amount of time they practiced using each method of training, depending on their randomized group allocation.

\section{Posttest}

The posttest sessions were identical to the pretest sessions, with the same volunteer and expert evaluator present. The student performed an RECD measurement by placing the probe tube and foam tip, followed by completing the same questionnaire as the pretest. On completion of the test sessions and training period, the expert evaluator performed two RECD measurements on the volunteer to be averaged for a gold standard comparison.

\section{Student Questionnaire}

The student questionnaire aimed to examine each participant's level of self-efficacy in four areas: (a) placement of the probe tube within $5 \mathrm{~mm}$ of the TM; (b) placement of the probe tube without contact with the TM; (c) placement of a foam tip after probe-tube placement such that the probe tube did not move (closer to, or further from, the TM); and (d) self-efficacy in placing a probe tube in a clinical setting (Table 1, sections A and B). The survey questions consisted of Likert scale responses that ranged in $10 \%$ increments from $0 \%$ to $100 \%(0 \%=$ "cannot do at all"; $50 \%=$ "sometimes can do"; and $100 \%$ = "always can do").

\section{Expert Evaluator Questionnaire}

The expert evaluator questionnaire aimed to measure how the evaluator perceived the participant's ability to conduct most aspects of the RECD measurements and key factors that lead to a successful/unsuccessful execution in clinic (Table 1, section C). The items were similar to the aspects of probe-tube placement and RECD measurement the participants would be evaluated on during their course practical examination. The survey questions consisted of a rating scale in $10 \%$ increments from $0 \%$ to $100 \%$ ( $0 \%$ = "strong disagreement"; $50 \%=$ "neither agree nor disagree"; and $100 \%=$ "strong agreement").

\section{Statistical Analysis}

Data analysis conducted on the questionnaire data used a Wilcoxon $t$-test to determine significance within groups to compare pre- versus posttest results and a Mann-Whitney $\mathrm{U} t$-test to test significance for group differences (simulator versus control group), in which a significance of $p<0.01$ was chosen. A two-way mixed analysis of variance (ANOVA) was used to examine time effects between pre- and posttests, group effects between the simulator and control groups, and interactions to determine whether they were present for all of the measures. A value of $p<0.05$ was chosen for the ANOVA.

For the RECD measurements, the average level of the frequency-specific RECD values were reduced to three bands for analysis to represent the frequency ranges that are affected by venting in the low frequencies, probe placement in the high frequencies, and the mid-frequency range in between. These were low frequency $(200-945 \mathrm{~Hz})$, mid frequency $(1000-2800 \mathrm{~Hz})$, and high frequency $(3000-8000 \mathrm{~Hz}$ ) (Dirks et al, 1996). A nonlinear mixed model statistical method was used for analysis using the RECD value, treatment, session, and frequency, in which a significance of $p<$ 0.05 was considered. Means within each frequency band were calculated and descriptively compared with a gold standard measurement (expert evaluator's 
measurements of the RECD) for both pre- and posttest measurements of each group.

\section{RESULTS}

\section{Overview}

Results from the questionnaire and RECD measurements are presented in the following sections: pretest simulator versus pretest control, comparison of training times for each group, posttest simulator versus posttest control, and pretest versus posttest for the simulator and control groups.

\section{Pretest Comparison Results}

All questionnaire pretest results from both groups were not significantly different, and all RECD measurements between the two groups were not significantly different.

\section{Training Times}

The control group practiced using traditional methods for $115 \mathrm{~min}$ (standard deviation [SD] $=71 \mathrm{~min}$ ), whereas the simulator group practiced with traditional methods for $98 \mathrm{~min}(\mathrm{SD}=53 \mathrm{~min}$ ) on average, but no significant difference was found between these two groups. The simulator group supplemented their traditional methods of training with $71 \mathrm{~min}(\mathrm{SD}=31)$ of simulator usage on average. The total training time for the simulator group was $169 \mathrm{~min}(\mathrm{SD}=58)$, showing a significant difference between the control group's total training time of $115 \mathrm{~min}(\mathrm{SD}=71, p=0.036)$.

\section{Posttest Comparison Results}

All questionnaire posttest results between the two groups were not significantly different. RECD measurements between groups in the posttest session were not significantly different in any bands.

\section{Pre- Versus Posttest Results: Questionnaire}

Three questions (questions 1, 3, and 5) out of ten revealed significantly improved results for the simulator and control group. Three other questions (questions 2, 8, and 9) showed significantly improved results for only the simulator group ( $p=0.008$, 0.008 , and 0.008 ), suggesting that the use of the simulator produced improved confidence in probe-tube placement without contacting the TM, improved usage of the foam tip, and increased perceived confidence (Figure 3). The remaining four questions (questions $4,6,7$, and 9) showed nonsignificant improvements for both groups.
The two-way mixed ANOVA revealed no significant group effect, a consistent time effect for each question, and interactions for questions 3 and 9 ( $p=0.049$ and 0.040 , respectively). The interactions can be seen in Figure 3 , with the simulator group showing notable improvement in placing the foam tip without affecting the probe and perceived confidence.

\section{Pre- Versus Posttest Results: RECD Measurements}

In the RECD results, it was found that the high-, mid-, and low-frequency bands of the simulator group all showed a significant effect of the simulator use on the RECD values $\left(p<0.001, \chi^{2}=36.9 ; p=0.004, \chi^{2}=\right.$ 8.1; $p<0.001, \chi^{2}=29.6$, respectively). An ANOVA was used to analyze the difference between pretest RECD and expert RECD at each frequency and the difference between posttest RECD and expert RECD at each frequency. The statistical analysis showed that, in the simulator group, posttest results were significantly closer to the expert's RECD measurements for the high $(F=10.34, p=0.00152)$, mid $(F=5.045, p=0.0258)$, and low $\left(F=137.6, p=2 \times 10^{-16}\right)$ frequency bands. In the control group, only the high-frequency band showed a significant effect from the training period on the RECD values ( $p=0.003, \chi^{2}=8.9$ ). When comparing to the expert's measurements, it was found that in the mid-frequency band, the RECD average of the pretest was statistically closer to the expert RECDs than the posttests $(F=5.238, p=0.0229$ ), suggesting the pretest measurements were statistically more accurate. Conversely, in the low-frequency band, posttest measurements were found to be statistically closer to the expert measurements $\left(F=390.8, p=2 \times 10^{-16}\right)$ (Figure 4).

\section{DISCUSSION}

I $\mathrm{n}$ medical simulator research, a typical validation sequence consists of a qualitative study aiming to receive early-stage feedback (face and content validity), backed with quantitative studies to prove its utility within educational settings (construct validity, discriminant validity, and skill transference validity). Our previous study (Koch et al, 2018) confirmed the simulator's training ability and provided feedback to guide the product development discussed previously. The present study accomplished a second-level evaluation of the developed audiology training simulator. Whereas the first-level face and content validity study previously performed on an early prototype of the simulator recruited experts to evaluate the simulator, the present study required novice students to observe skill progression throughout the duration of the study. Completion of the study within the student's course load introduced challenges. First, the time frame of the study was very limited. Students in the audiology program at Western 

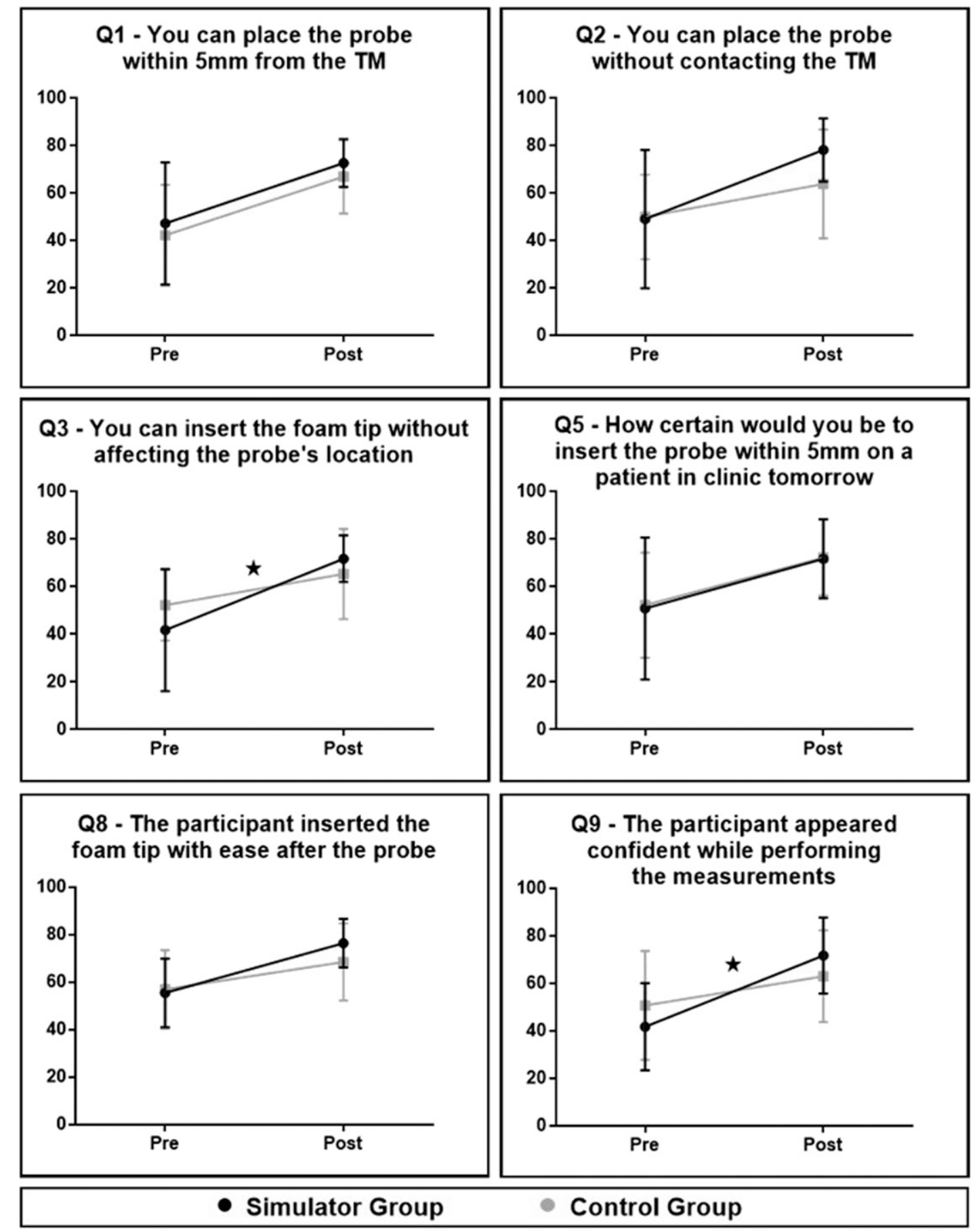

Figure 3. ANOVA results from the questionnaire results. Questions 1, 3, and 10 produced significant differences between pre- and posttest results for both groups. Questions 2, 6, and 7 produced significant differences between pre- and posttests for the simulator group but not the control group. ANOVA interactions were found in questions 3 and 7 (noted by the asterisk). All other questions showed no significant differences.

University have a practical examination in probe-tube placement one month after their first probe-tube placement laboratory session. Because of this schedule, the training period of this study was limited to two weeks to guarantee the control group equal opportunity to use the simulator after the completion of this study but before their practical examination as required by Health Research Ethics Board. Second, the preand posttest sessions were limited to one probe-tube placement to work within the time frame of the study and to accurately replicate a clinical scenario in which students would have time for one placement. Despite these limitations, the study found several meaningful results. 

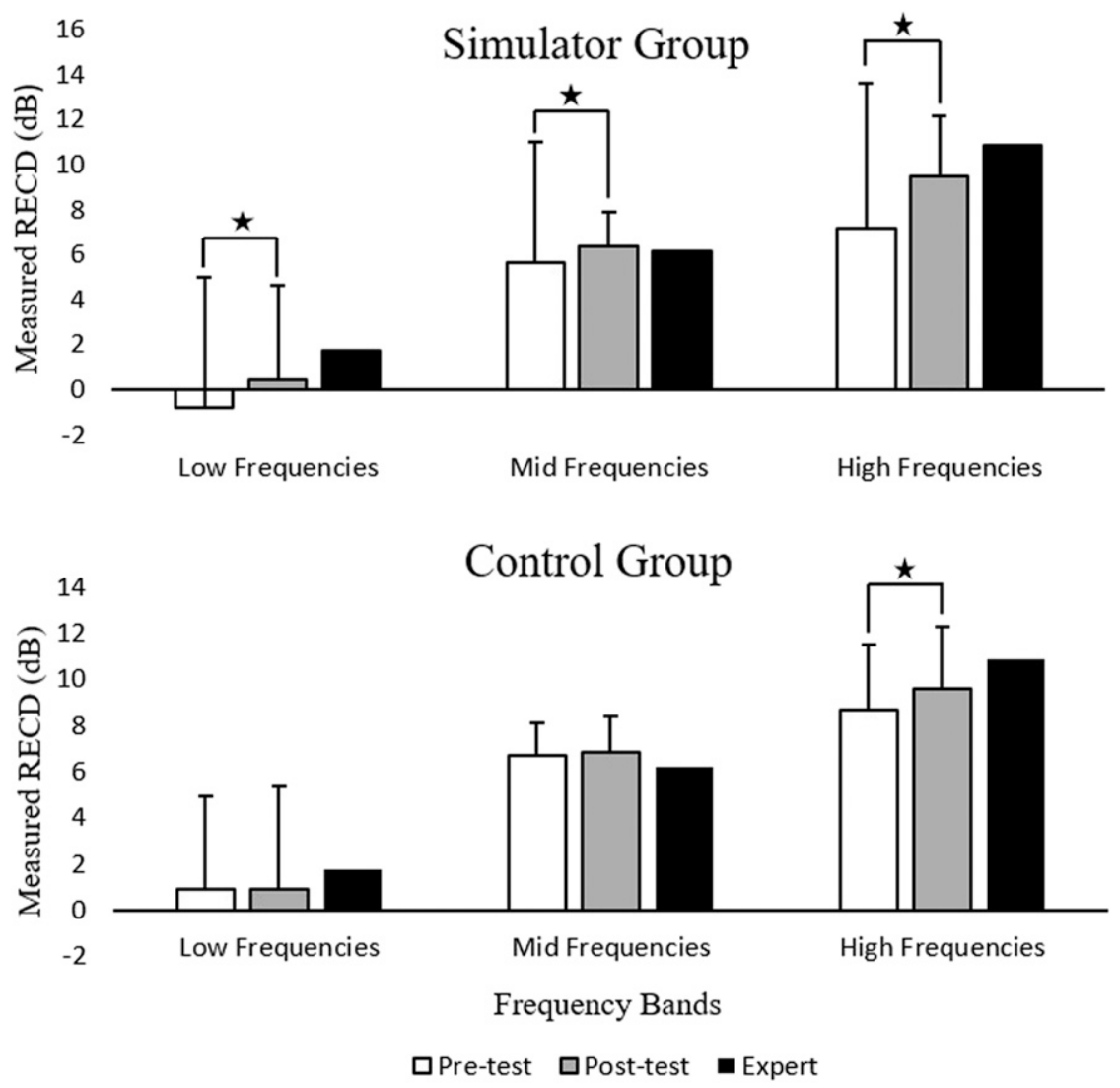

Figure 4. Bar graph displaying the means of the RECD measurements made by the simulator group (top) and control group (bottom) in the pre- and posttests. Results are averaged across three bands: low, medium, and high frequencies (see text for details). Measurements with a significant difference between the two sessions are marked with an asterisk. Error bars denote one SD.

No significant pretest differences were found in either the questionnaire or the RECD results, suggesting that both the simulator and control group had equivalent skill levels at the beginning of this study. It should be noted that the pretest self-assessment results had a large amount of variation, showing that each student started with their own level of confidence even though they each had roughly the same practice time. This high level of confidence in the pretest session did not necessarily correlate with a highly accurate RECD measurement. No significant posttest differences were found, and there were no significant improvements when only comparing posttest results.

There were several notable differences between the pre- and posttest session questionnaires and the realear measures. First, question 2 (self-evaluation for "You can place the probe without contacting the TM") produced a significant improvement for the simulator group but not for the control group. This result along with the positive trend in the ANOVA for the same question (Figure 3) suggests the simulator's feedback mechanisms of contacting the TM and providing users insight into where the TM is located in the simulated ear canal may have an impact on the clinical performance of their probe-tube placement. Recall that the control group practiced on one another and were likely motivated to avoid contact with the TM during practice. With the simulator, it is feasible to practice intentionally by placing the probe tube too far to learn how to avoid this without fear of an aversive experience for one's classmate. This study only measured the time span of student practice with the simulator, so we have not directly assessed whether this factor is important for students' perceived confidence in not striking the TM. High-frequency similarities in the RECD measures may also suggest that the probe depth is comparable between the two groups, but with the simulator group being less likely to contact the TM, which is an important aspect of clinical probe-tube placement.

Second, several results suggest that participants who used the simulator had better usage of the foam tip to occlude the ear canal. Question 3 (self-evaluation of "You can insert the foam tip without affecting the probe's location") produced an interaction in the ANOVA, whereas the expert's evaluation of the participant easily inserting the foam tip (question 8) produced significantly improved results for the simulator group, but not for the control group. The simulator's ability to provide real-time feedback on the probe's position while inserting the foam tip may allow for users to be more 
aware of these changes in a clinical scenario. The RECD measurements also reiterate that the foam tip may have better occluded the ear canal within the simulator group than the control group, as significant changes were found in the low-frequency band of only the simulator group. Again, this reflects a better overall level of competency with the details of the measurement procedure.

Third, the ANOVA presented interactions between the two groups in participants' confidence to place the foam tip without affecting the probe (question 3) and participants' perceived confidence (question 9). Confidence is extremely important in clinic, with studies showing that clinician confidence, patient's perception, and trust of the clinician influence hearing outcomes (Amlani et al, 2016; 2017). A new clinician entering clinic with improved confidence may not only deliver better care to the patient but also improve the patient's trust. This may also relate to the issues around additional practice noted previously.

Finally, the RECD results reinforced the questionnaire results. The significant improvements found within the RECD measurements in the simulator group suggest more improved measurements were taken in the posttest scenario for the simulator group. The improved occlusion of the ear canal with the foam tip may be related to the probe-tip insertion depth suggested by the RECD. The control group's higher propensity to touch the TM with the probe, along with the similar high-frequency RECD values, suggests a deeper insertion depth than the simulator group.

These positive results are also present with a nonsignificant difference between traditional training times. Recall that the simulator group only supplemented their training with the simulator, so this overall improvement in competency may reflect additional practice time. Although this may or may not be attributable to the simulator itself (i.e., perhaps more traditional practice could have achieved the same result), we note that additional practice via the simulator does not require a laboratory partner and, therefore, may support flexibility and independence in performing additional practice sessions while learning key procedures.

As the simulator is still a working prototype, final feedback was received from the participants in the form of written recommendations. Participants noted the most beneficial parts of the simulator for probe-tube placement were (a) the ability to practice on their own, (b) the ability to know how deep they were placing the probe tube, and (c) the ability to know exactly how to judge when the probe tube was placed within $5 \mathrm{~mm}$ of the TM.

Next steps for this project include continued improvement to the simulator. As development on the simulator evolves, more ear anatomies will be available (e.g., large ear canal, small ear canal, pediatric ears, exostosis, and mastoid cavity) and specific training use cases for the simulator will be created. With these results showing encouraging effects of simulator use in clinical programs, there are several other procedures which still cannot be simulated in an educational setting. Initial tests have been performed with earmold impressions and the insertion of RICs and earmolds into the canal with the algorithm providing feedback to the location of the mold, the RIC tip or the otoblock in the canal. Additional work will be put toward incorporating this into the design and ensuring the clinical training needs are met.

\section{CONCLUSION}

$\mathrm{I}$ $\mathrm{n}$ conclusion, this study has found benefits to trainees' usage of the probe-tube placement simulator. Results suggest that students who supplemented their traditional training with the simulator were less likely to contact the TM in a clinical scenario, more likely to perform a better ear canal occlusion resulting in improved RECD measurements in the low frequencies, more likely to achieve appropriate probe-tube placement with improved RECD measurement in the high frequencies, and more likely to appear confident. With two validation studies completed, future work will aim to address final concerns and the initial supplying of this simulator system to training programs to improve trainee performance while decreasing the workload on instructors.

\section{REFERENCES}

Aazh H, Moore BCJ. (2007) The value of routine real ear measurement of the gain of digital hearing aids. J Am Acad Audiol 18: 653-664.

Aazh H, Moore BCJ, Prasher D. (2012) The accuracy of matching target insertion gains with open-fit hearing aids. Am J Audiol 21: $175-180$.

Abrams HB, Chisolm TH, McManus M, McArdle R. (2012) Initialfit approach versus verified prescription: comparing self-perceived hearing aid benefit. J Am Acad Audiol 23:768-778.

Amlani AM, Pumford J, Gessling E. (2016) Improving patient perception of clinical services through real-ear measurements. Hear Rev. http://www.hearingreview.com/2016/11/improving-patientperception-clinical-services-real-ear-measurements/. Accessed June 14, 2018.

Amlani AM, Pumford J, Gessling E. (2017) Real-ear measurement and its impact on aided audibility and patient loyalty. Hear Rev. http://www.hearingreview.com/2017/09/real-ear-measurementimpact-aided-audibility-patient-loyalty/. Accessed June 14, 2018.

Anderson MC, Arehart KH, Souza PE. (2018) Survey of current practice in the fitting and fine-tuning of common signal-processing features in hearing aids for adults. J Am Acad Audiol 29:118-124.

Bagatto MP, Seewald RC, Scollie SD, Tharpe AM. (2006) Evaluation of a probe-tube insertion technique for measuring the realear-to-coupler difference (RECD) in young infants. J Am Acad Audiol 17:573-581. 
Bamford J, Beresford D, Mencher G, DeVoe S, Owen V, Davis A. (2001) Provision and fitting of new technology hearing aids: implications from a survey of some "good practice services" in UK and USA. In: In: Seewald RC, Gravel JS, eds. A Sound Foundation through Early Amplification: Proceedings of an International Conference. Stafa, Switzerland: Phonak AG, 213e219.

British Society of Audiology (BSA). (2007) Guidance on the use of real ear measurement to verify the fitting of digital signal processing hearing aids. Br Soc Audiol 1:1-20.

Brown DK. (2017) Simulation before clinical practice: the educational advantages. Audiology Today 29:16-24.

Caldwell M, Souza PE, Tremblay KL. (2006) Effect of probe tube insertion depth on spectral measures of speech. Trends Amplif 10: $145-154$

College of Audiologists and Speech-Language Pathologists of Ontario (CASLPO). (2016) Practice standards for the provision of hearing aid services by audiologists. Toronto, ON: CASLPO. http://www.caslpo.com/ sites/default/uploads/files/PS_EN_Practice_Standards_for_the_Provision_ of_Hearing_Aid_Services_By_Audiologists.pdf.

Consumer Reports. (2009) Hearing Aid Shoppers Pay High Prices, Get Mediocre Fittings. Consumer Reports. New York, NY: Consumers Union. https://www.consumerreports.org/media-room/ press-releases/2009/06/hearing-aid-shoppers-pay-high-prices-getmediocre-fittings- $/$.

Dawe SR, Pena GN, Windsor JA, Broeders JA, Cregan PC, Hewett PJ, Maddern GJ. (2014) Systematic review of skills transfer after surgical simulation-based training. $\mathrm{Br} J$ Surg 101:1063-1076.

Dirks DD, Ahlstrom JB, Eisenberg LS. (1996) Comparison of probe insertion methods on estimates of ear canal SPL. J Am Acad Audiol 7:31-38.

Dirks DD, Kincaid GE. (1987) Basic acoustic considerations of ear canal probe measurements. Ear Hear 8:60S-67S.

Gaba DM. (2004) The future vision of simulation in health care. Qual Saf Heal Care 13:2-10.

Huang C, Cheng H, Bureau Y, Agrawal SK, Ladak HM. (2015) Face and content validity of a virtual-reality simulator for myringotomy with tube placement. J Otolaryngol Head Neck Surg 44:1-8.

ISO. (2001) ISO 12124:2001 - Acoustics- Procedures for the Measurement of Real-Ear Acoustical Characteristics of Hearing Aids. Geneva, Switzerland: ISO.

Issenberg SB, McGaghie WC, Hart IR, Mayer JW, Felner JM, Petrusa ER, Waugh RA, Brown DD, Safford RR, Gessner IH, Gordon DL, Ewy GA. (1999) Simulation technology for health care professional skills training and assessment. JAMA 282: 861.

Jorgensen LE. (2016) Verification and validation of hearing aids: opportunity not an obstacle. J Otol 11:57-62.

Koch RW, Moodie S, Folkeard P, Scollie S, Janeteas C, Agrawal SK, Ladak HM. (2018) Face and content validity of a probe tube placement training simulator. J Am Acad Audiol 30(3):227-234.

Kunkler K. (2006) The role of medical simulation: an overview. Int J Med Robot Comput Assist Surg 2:203-210.
Laschinger S, Medves J, Pulling C, McGraw DR, Waytuck B, Harrison MB, Gambeta K. (2008) Effectiveness of simulation on health profession students' knowledge, skills, confidence and satisfaction. Int $J$ Evid Based Healthc 6 : 278-302.

Leavitt R, Bentler R, Flexer C. (2017) Hearing aid programming practices in Oregon: fitting errors and real ear measurements. The Hearing Rev 24:30-33.

Leavitt RJ, Flexer C. (2012) The importance of audibility in successful amplification of hearing loss. Hear Rev 19(13):20e23.

McCreery RW, Bentler RA, Roush PA. (2013) Characteristics of hearing aid fittings in infants and young children. Ear Hear 34: 701-710.

Moodie S, Rall E, Eiten L, Lindley G, Gordey D, Davidson L, Bagatto M, Scollie S. (2016) Pediatric audiology in North America: current clinical practice and how it relates to the American Academy of Audiology Pediatric Amplification Guideline. J Am Acad Audiol 27:166-187.

Moodie S, Pietrobon J, Rall E, Lindley G, Eiten L, Gordey D, Davidson L, Moodie KS, Bagatto M, Haluschak MM, Folkeard P, Scollie S. (2016) Using the real-ear-to-coupler difference within the American Academy of Audiology Pediatric Amplification Guideline: protocols for applying and predicting earmold RECDs. $J$ Am Acad Audiol 27:264-275.

Mueller HG. (2005) Probe-mic measures: hearing aid fitting's most neglected element. Hear $J$ 58:1-6.

Mueller HG, Picou E. (2010) Survey examines popularity of realear probe-microphone measures. Hear $J$ 63:27-32.

OpenCV Team. (2018) OpenCV Homepage. https://opencv.org/. Accessed July 4, 2018.

Palmer CV. (1998) Curriculum for graduate courses in amplification. Trends Amplif 3:6-44.

Pumford J, Sinclair S. (2001) Real-ear measurement: basic terminology and procedures. Audiol Online. http://www.audiologyonline. com/articles/real-ear-measurement-basic-terminology-1229. Accessed April 24, 2018.

Torkington J, Smith SGT, Rees BI, Darzi A. (2001) Skill transfer from virtual reality to a real laparoscopic task. Surg Endosc 15: 1076-1079.

Vaisberg JM, Macpherson EA, Scollie SD. (2016) Extended bandwidth real-ear measurement accuracy and repeatability to $10 \mathrm{kHz}$. Int $J$ Audiol 55:1-7.

Valente M. (2006) Guideline for Audiologic Management of the Adult Patient. Audiol Online. https://www.audiologyonline.com/ articles/guideline-for-audiologic-management-adult-966. Accessed June 10, 2018.

Valente M, Odeing K, Brockmeyer A, Smith S, Kallogjeri D. (2017) Differences in word and phoneme recognition in quiet, sentence recognition in noise, and subjective outcomes between manufacturer first-fit and hearing aids programmed to NAL-NL2 using real-ear measures. J Am Acad Audiol 29:706-721.

Wang AT, Erwin PJ, Hamstra SJ. (2011) Technology-enhanced simulation. J Am Med Assoc 306:978-988. 